\title{
Genomic insights of protein arginine methyltransferase Hmt1 binding reveals novel regulatory functions
}

Eric J Milliman ${ }^{1}$, Zihua $\mathrm{Hu}^{2,3}$ and Michael C $\mathrm{Yu}^{1 *}$

\begin{abstract}
Background: Protein arginine methylation is a post-translational modification involved in important biological processes such as transcription and RNA processing. This modification is catalyzed by both type I and II protein arginine methyltransferases (PRMTs). One of the most conserved type I PRMTs is PRMT1, the homolog of which is Hmt1 in Saccharomyces cerevisiae. Hmt1 has been shown to play a role in various gene expression steps, such as promoting the dynamics of messenger ribonucleoprotein particle (mRNP) biogenesis, pre-mRNA splicing, and silencing of chromatin. To determine the full extent of Hmt1's involvement during gene expression, we carried out a genome-wide location analysis for Hmt1.

Results: A comprehensive genome-wide binding profile for Hmt1 was obtained by ChIP-chip using NimbleGen high-resolution tiling microarrays. Of the approximately $1000 \mathrm{Hmt1-binding} \mathrm{sites} \mathrm{found,} \mathrm{the} \mathrm{majority} \mathrm{fall} \mathrm{within} \mathrm{or}$ proximal to an ORF. Different occupancy patterns of Hmt1 across genes with different transcriptional rates were found. Interestingly, Hmt1 occupancy is found at a number of other genomic features such as tRNA and snoRNA genes, thereby implicating a regulatory role in the biogenesis of these non-coding RNAs. RNA hybridization analysis shows that Hmt1 loss-of-function mutants display higher steady-state tRNA abundance relative to the wild-type. Co-immunoprecipitation studies demonstrate that Hmt1 interacts with the TFIIIB component Bdp1, suggesting a mechanism for Hmt1 in modulating RNA Pol III transcription to regulate tRNA production.

Conclusions: The genome-wide binding profile of Hmt1 reveals multiple potential new roles for Hmt1 in the control of eukaryotic gene expression, especially in the realm of non-coding RNAs. The data obtained here will provide an important blueprint for future mechanistic studies on the described occupancy relationship for genomic features bound by Hmt1.
\end{abstract}

Keywords: Protein arginine methylation, Hmt1, RNA Pol III transcription, tRNA biogenesis, ChIP-chip

\section{Background}

Protein arginine methylation is a post-translational modification commonly found in nucleic acid-binding proteins [reviewed in [1,2]. This modification is catalyzed by a family of evolutionarily conserved enzymes called protein arginine methyltransferases (PRMTs). PRMTs can be classified into four major classes depending on the type of methylarginine generated by the enzyme. Type I, II, and III PRMTs can all catalyze monomethylation and generate monomethylarginine (MMA) on a guanidinum nitrogen of arginine residues

\footnotetext{
* Correspondence: mcyu@buffalo.edu

'Department of Biological Sciences, State University of New York at Buffalo, Buffalo, NY 14260, USA

Full list of author information is available at the end of the article
}

in proteins. Type I PRMTs then catalyze the formation of asymmetric dimethylarginine (ADMA) and type II PRMTs, symmetric dimethylarginine (SDMA). Type III can only catalyze monomethylarginine while the Type IV enzyme, which has only been described in Saccharomyces cerevisiae [3], catalyzes monomethylation of the delta nitrogen atom of the arginine residue. Currently, eleven PRMT family members have been identified in the metazoans, with PRMT1 (Type I) and PRMT5 (Type II) being the most conserved members of the PRMT family across the eukaryotic spectrum reviewed in [4].

Hmt1 (also termed Rmt1) has been identified as the major type I PRMT in the budding yeast $S$. cerevisiae and is the functional homolog of mammalian PRMT1 [5,6]. A 
number of substrates have been identified for Hmt1 and knowledge of these substrates' biological functions has helped infer Hmt1's role as a regulator during various steps in gene expression. These substrates include histones $\mathrm{H} 2 \mathrm{~A}, \mathrm{H} 2 \mathrm{~B}, \mathrm{H} 3$, and $\mathrm{H} 4$ [7,8], mRNA export factors Npl3 [5] and Yra1 [9], pre-mRNA splicing factors Snp1 [10], 3'end processing factors Hrp1 [11] and Nab2 [12], and the nucleolar proteins Nop1, Nsr1, and Gar1 [13]. Functional studies have shown that yeast mutants lacking Hmt1 display compromised intracellular protein trafficking, aberrant messenger ribonucleoprotein particle (mRNP) formation and defective silent chromatin formation [9,11,14]. The defects seen in the silent chromatin formation in Hmt1 loss-of-function mutants may be due to a change in the recruitment of other histone deacetylases to these regions, thereby altering the histone modification status at such regions [15]. Moreover, recent data from large-scale, synthetic genetic array (SGA) experiments reveal new, potential functions for Hmt1 in a cell [15-17].

Genome-wide location analysis is a powerful method that allows one to comprehensively identify direct and indirect protein-nucleic acid interactions across a specific genome in vivo, by combining the method of chromatin immunoprecipitation followed by hybridization to microarrays reviewed in $[18,19]$. For yeast, this approach has been utilized to study both DNA- and RNA-binding proteins [20-23]. For example, genome-wide location analyses of basal transcription factors have provided important information on the marking of promoter regions within a eukaryotic transcription circuit [24,25]. Additionally, the ability to use antibodies directed against specific histone modifications has enabled the creation of chromatin signature maps marking promoters that are either active or repressed, as well as other genome elements such as enhancers and transcribed regions reviewed in [26]. Thus, this approach has helped answer important questions about where in the genome a protein physically interacts and can unravel critical details of gene regulation within a cell.

In this study, we have comprehensively mapped the genome-wide occupancy for Hmtl using NimbleGen high-resolution tiling microarrays. Based on the profile of genomic features bound by Hmt1, we found that Hmt1binding sites are located at a number of ORFs as well as noncoding RNA (ncRNA) genes such as tRNA and snoRNA genes. To probe the potential functional consequence of Hmt1-occupancy at tRNA genes, we assayed the steady-state levels of various tRNAs in Hmt1 loss-offunction mutants and found their levels to be elevated when compared to wild-type cells. This positive change in the tRNA abundance in the Hmt1 loss-of-function mutants is likely due to a changed transcriptional output by RNA polymerase III (RNAPIII) rather than a change in the tRNA maturation process. Lastly, coimmunoprecipitation experiments show an association between Hmt1 and the TFIIIB component Bdp1, which identifies a potential molecular link by which Hmt1 is recruited to tRNA genes. Together, these results provide a global view for Hmt1 function within the cell and reveal a novel role for Hmt1 in regulating tRNA biogenesis.

\section{Results}

\section{Yeast genomic features bound by Hmt 1}

Hmt1 has been previously shown to be cotranscriptionally recruited to genes [9] and Hmt1 loss-of-function mutants display decreased levels of dimethylated arginine 3 of histone H4 (H4R3) at silent chromatin regions [14]. Together, these observations demonstrate the significance of Hmt1's interaction with the genome in facilitating its biological roles within a cell. While we have previously carried out genome-wide location analysis for Hmt1 in the past [9], that experiment used cDNA microarrays. Thus, there remains a lack of knowledge with respect to other genomic features that Hmt1 may interact with, such as autonomous replicating sequences (ARSs) or non-coding RNA (ncRNA) genes, which are not represented on the cDNA microarray platforms. To address this gap in knowledge for Hmt1's association with the genome, we have comprehensively mapped the genome-wide occupancy of Hmt1 using NimbleGen's high-resolution yeast tiling microarrays. These microarrays gave us the resolution and coverage needed for fully assess Hmtl's genome-wide occupancy. After acquiring the data, ACME peak finding was used to analyze individual microarray data sets. We then used custom PERL scripts to integrate our biological triplicate datasets and to identify Hmt1-binding sites. Using this information, we were able to identify genomic features that are enriched for Hmt1-binding sites in our data sets and extracted peak sequences for analyses such as motif discovery.

Overall, a total of $1012 \mathrm{Hmt1}$ binding sites (with a peak size of $250 \mathrm{bp}$ ) were found to have passed the cutoff criteria for statistical significance (see Additional file 1: Table S1). Approximately $71 \%$ of these binding sites map to regions within or proximal to an annotated ORF (within $125 \mathrm{bp}$ of the peak mid-point) (Figure 1A). However, this represents only $10 \%$ of all ORFs within the genome (Figure 1B). Using Gene Ontology enrichment analysis [27], we found that ORFs bound by Hmt1 are enriched for genes involved in translation (such as translational elongation, ribosomal protein genes, cytosolic ribosome, etc) and in rRNA maturation pathways (Table 1 ). Hmt1-binding sites also map to a number of ncRNA genes, including tRNAs, snRNAs, and snoRNAs, as well as ARSs (Figure 1A). The percentage of total features within the yeast genome that are bound by Hmt1 was calculated for each of the annotated genomic element classes (Figure 1B). This analysis revealed that Hmt1 binds to approximately $40 \%$ of all nuclear-encoded tRNA genes, as well as nearly $35 \%$ of all snoRNAs. 

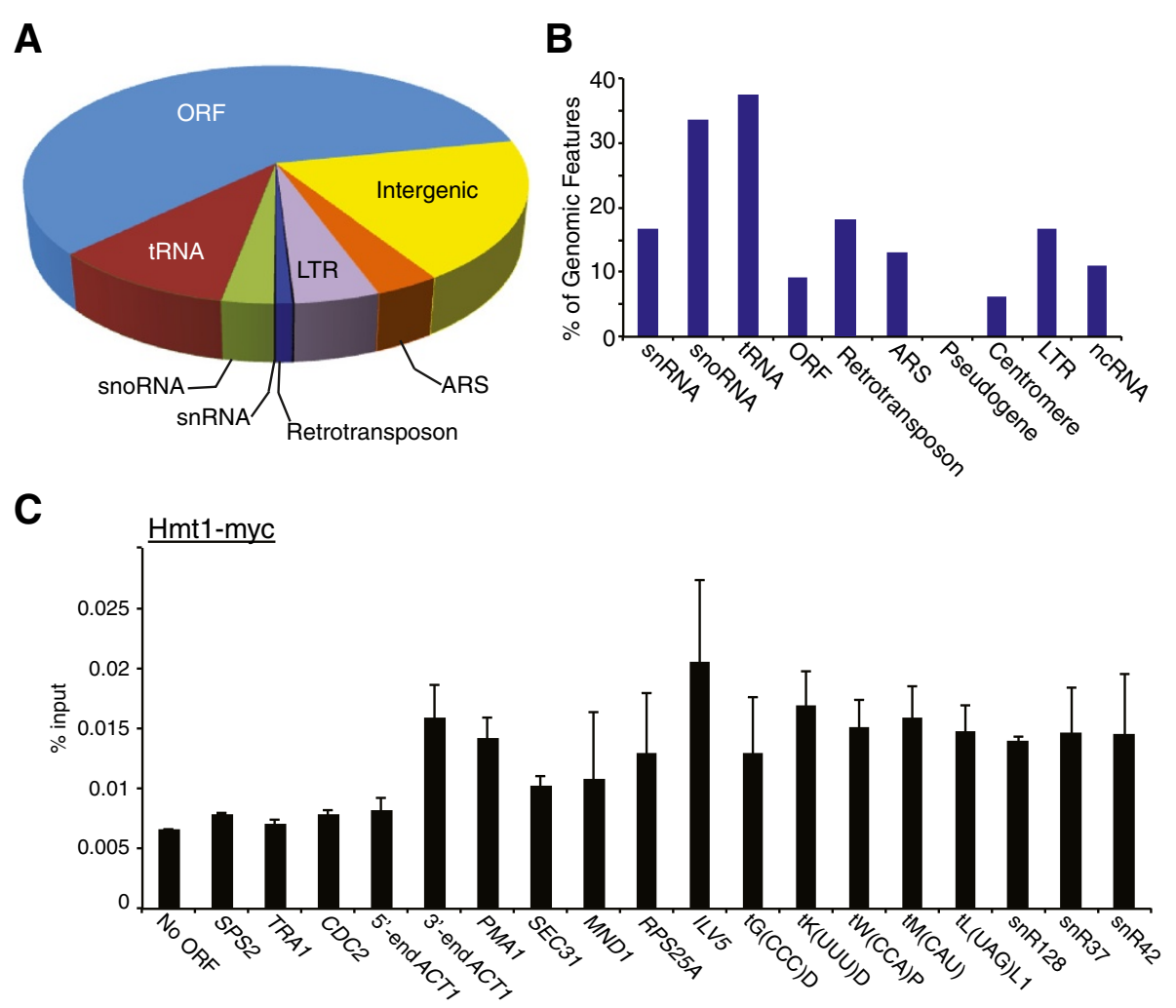

Figure 1 Genome-wide binding profiles of Hmt1. A) Pie chart showing the fraction of Hmt1 binding sites that mapped to the indicated genomic feature. B) The percentage of each genomic feature bound by Hmt1 as compared to the total number of that feature in the nuclear genome. C) Directed ChIP validation of genes bound by Hmt1. ChIP signals were normalized to "No ORF", which is an intergenic region on the right arm of Chromosome IV [68]. TRA1, CDC2, and SPS2 were negative controls. The use of ACT1 was to demonstrate the enrichment of Hmt1 occupancy within specific regions of a gene body. Lastly, a number of tDNAs binding sites were confirmed.

Table $1 \mathrm{GO}$ term analysis for gene functional groups enriched in Hmt1-binding sites

\begin{tabular}{lll}
\hline Adj. $\boldsymbol{p}$-value & GO attribute & Description \\
\hline$<0.001$ & 6414 & translational elongation/protein synthesis elongation \\
$<0.001$ & 30533 & triplet codon-amino acid adaptor activity/tRNA/transfer RNA \\
$<0.001$ & 16283 & eukaryotic 48S initiation complex \\
$<0.001$ & 16072 & rRNA metabolism \\
$<0.001$ & 16282 & eukaryotic 43S preinitiation complex/eukaryotic 43S \\
$<0.001$ & 9451 & RNA modification \\
$<0.001$ & 5843 & cytosolic small ribosomal subunit/40S ribosomal subunit/cytosolic small ribosomal subunit \\
$<0.001$ & 30563 & snRNA 2' -O- ribose methylation guide activity \\
$<0.001$ & 17069 & snRNA binding \\
$<0.001$ & 30561 & RNA 2' -O- ribose methylation guide activity \\
$<0.001$ & 30566 & snRNA modification guide activity \\
$<0.001$ & 154 & rRNA modification \\
$<0.001$ & 5842 & cytosolic large ribosomal subunit/60S ribosomal subunit/cytosolic large ribosomal subunit \\
$<0.001$ & 30555 & RNA modification guide activity \\
$<0.001$ & 30529 & ribonucleoprotein complex/RNP \\
\hline
\end{tabular}


To validate our high-resolution ChIP-chip data of Hmt1, we used directed ChIP (ChIP-qPCR) to verify a number of the identified binding sites. In each case, we have identified Hmt1 enrichment at the regions tested (Figure 1C). These features include specific regions within a few ORFs and ncRNA genes. As a negative control, we measured Hmt1 enrichment at regions not found in our Hmt1-bound dataset, such as TRA1 and SPS2 (Figure 1C). The results from our ChIP validation experiments provide additional confidence that $\mathrm{Hmt1}$ binds a number of genomic features previously unknown, especially tRNA genes.

\section{Characteristics of Hmt1-binding across ORFs}

Previously, we have shown that Hmt1 is cotranscriptionally recruited to the actively transcribing gene GAL10, with a preference at the 5'-end to the middle of the GAL10 ORF [9]. To examine the relationship between Hmt1 occupancy and gene expression, we assigned ORF-encoding genes to three different classes based on their transcriptional
A
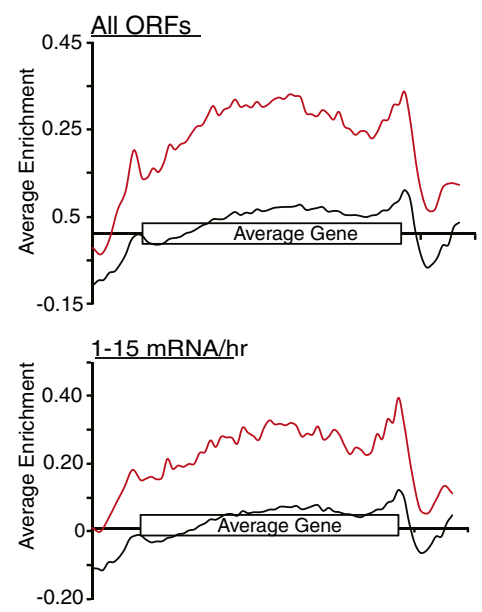

B

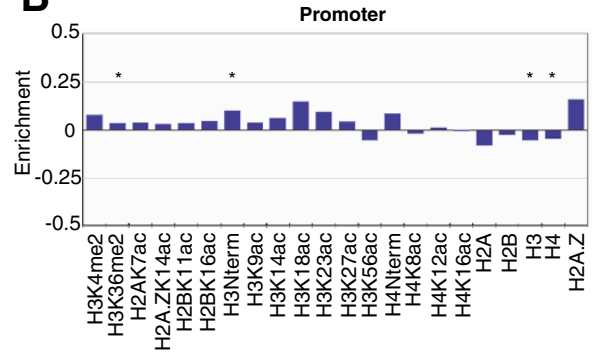

C

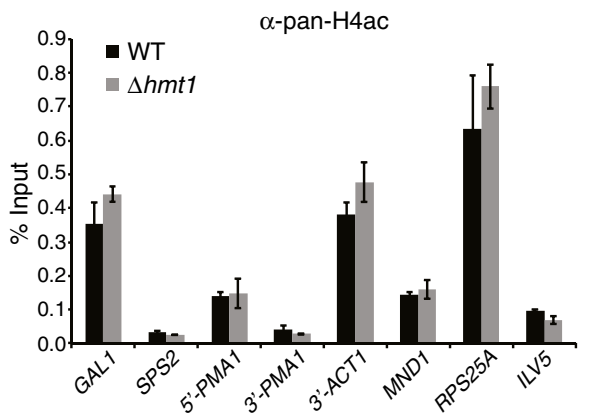

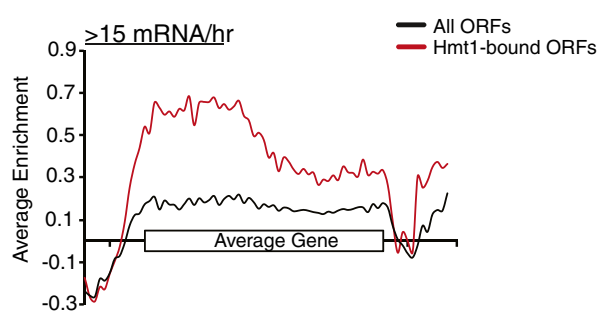
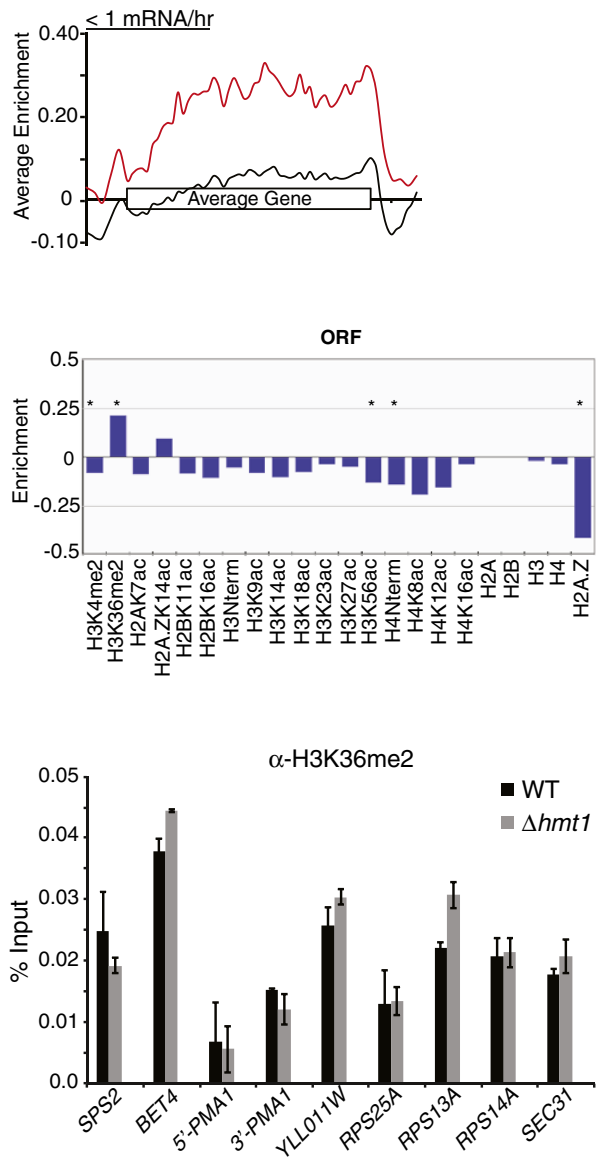

Figure 2 Hmt1-binding sites are enriched at ORF genes. A) Average Hmt1 enrichment profiles for all ORFs bound ("ALL ORFs), ORFs with high- (" $\left.\geq 15 \mathrm{mRNA} / \mathrm{hr} r^{\prime}\right)$, medium- ("1-15 mRNA/hr"), and low- (" $\leq 1 \mathrm{mRNA} / \mathrm{hr}$ ") transcriptional frequencies. B) Histone enrichment/depletion calculations of promoters and genes for Hmt1-bound ORFs from ChromatinDB. C) Directed ChIP to assess the role of Hmt1 on the levels of panH4ac and H3K36me2. Representative ORFs bound-by Hmt1 were assayed for the level of the specified histone modification in WT and hmt1-null cells. 
frequency [28]. We generated a baseline Hmt1 signal for all genes that fall under each of the three different classes as described previously [29] (Figure 2A, black lines in each panel), then we plotted the average Hmt1-enrichment for the set of ORFs that we classified as bound by our binding site annotations (Figure 2A, red lines in each panel). For highly transcribed genes ( $\geq 15 \mathrm{mRNAs} / \mathrm{hr}$ ), such as those involved in translation or ribosomal protein genes, there is an obvious enrichment of Hmt1 from the 5'-end to the middle of these highly transcribed genes (Figure 2A, upper right panel, red line). This occupancy trend is similar to what we had previously observed for Hmt1 on the GAL1O gene [9]. To our surprise, we observed a different trend for Hmt1 occupancy across ORFs with medium and low transcriptional rates (Figure 2A, lower right and lower left panels). In general, Hmt1 occupancy is evenly distributed across an ORF within these two transcriptional classes, with the exception of a slightly higher occupancy at the 3'-end of these ORFs. This is more apparent for the gene class with medium transcriptional frequency ( 1 to 15 mRNAs/hr) (Figure 2A, lower right panel, gray line).

Hmt1 methylates arginine 3 on histone H4 (H4R3) in vitro [7] and mutants lacking Hmt1 or its catalytic activities display decreased levels of dimethylated H4R3 at silent chromatin regions [14]. Since mutants lacking Hmt1 do not show a significant decrease in bulk dimethylated H4R3 [7], it is possible that Hmt1's effects on chromatin may only occur within a specific chromosomal context, such as those in the silent chromatin regions. Nevertheless, PRMT1 (the mammalian homolog of Hmt1)-catalyzed H4R3 methylation has been demonstrated as a key, initial histone modification that is important for subsequent histone modifications [30]. As such, we wanted to determine if Hmt1-bound ORFs displayed enrichment or depletion for specific histone modifications. Using ChromatinDB, we calculated the enrichment or depletion of 17 different histone modifications and the five core histones for the Hmt1-bound ORFs (Figure 2B) [31]. ChromatinDB calculates the enrichment/depletion in gene bodies as well as promoter regions across the yeast genome [31]. Analysis of the Hmt1-bound genes revealed that promoter regions were enriched for $\mathrm{H} 3 \mathrm{~K} 36 \mathrm{me} 2$ and pan-H3 acetylation, while being depleted for $\mathrm{H} 3 / \mathrm{H} 4$ occupancy (Figure 2B, promoter panel). H3K4me2, pan-H3 acetylation, H3K56ac, and H2A. $\mathrm{Z}$ occupancy are depleted, and $\mathrm{H} 3 \mathrm{~K} 36 \mathrm{me} 2$ is enriched across gene bodies (Figure 2B, ORF panel).

To assess whether Hmt1 regulates the modification of histones based on our ChromatinDB analysis, we performed directed ChIP using antibodies against pan-acetyl H4 and H3K36me2 in wild-type and hmt1-null cells to measure the levels of these modifications in ORF-gene bodies since we find the majority of Hmt1 enrichment here (Figure 2A). We tested the ORFs that were bound by Hmt1 and representative of the histone modification trend (i.e. enriched/depleted for a specific modification) based on the database analysis. With respect to pan-acetyl $\mathrm{H} 4$, we do not observe any changes in the level between wildtype and hmt1-null cells (Figure 2C, $\alpha$-pan-H4ac panel). When we compared the directed ChIP results for H3K36me2 between wild-type and hmt1-null cells, we found the majority of the ORFs we tested do not change, but there was a slight increase in two loci BET4 and RPS13A (Figure 2C, $\alpha-\mathrm{H} 3 \mathrm{~K} 36 \mathrm{me} 2$ panel). These results suggest that the absence (in the case of pan$\mathrm{H} 4 \mathrm{ac}$ ) or presence (in the case of H3K36me2) of these histone modifications may regulate the recruitment of Hmt1 as opposed to Hmt1 regulating their levels in ORF gene bodies.

\section{Analysis of Hmt1-bound sequences for motif discovery}

Because we were able to obtain detailed sequence-specific information from the probes used in our high-resolution microarray, we applied a number of motif finding algorithms (AlignACE [32], MEME [33], WEEDER [34], and MDScan [35]) to determine whether there exists a motif within the Hmt1 binding sites. While our analyses revealed enriched motifs, none of these appear to be of obvious biological significance (data not shown). This was not surprising to us, as Hmt1 has no reported DNA binding activity and is likely to associate within the proximity of the DNA via its interaction with DNA-binding proteins. This type of indirect interaction likely will impede finding true motif signals. However, we also took a reciprocal approach in which we searched all of the Hmt1-binding sites for known transcription factor sequence motifs using previously generated data [25,36,37]. Using the $250 \mathrm{bp}$ window for Hmt1-binding sites in our search and a negative control of 10,000 randomized yeast genome sequences of the same size, we carried out an analysis for the enrichment of known TF binding sites within our Hmt1-binding sites. Our analyses did reveal a number of over-represented TFbinding sites within the Hmt1-binding sites that passed statistical significance cutoff (Additional files 2: Table S2). A few of the most enriched TF-binding sites were for transcription factors Cat8, Tbp1, Dal81, Ume6, and Mig1. Except for Tbp1, which is the general, TATA-binding protein that interacts with other factors to form the preinitiation complex at promoters [38], the other four transcription factors are all involved in specific metabolic pathways [39-41] or life cycle stages (such as Ume6) [42]. Thus, it is possible that Hmt1 functions together with these transcription factors to help regulate the expression of genes involved in these pathways.

\section{Hmt1-binding is enriched at regions proximal to tRNA genes}

From our ChIP-chip data, we found that Hmt1-binding is enriched across the 5'-regions that are proximal or within 
the gene body of the nuclear-encoded tRNA genes (Figure 3A). Unlike the data from our Hmt1-bound ORF analyses for genes with medium- and low-transcriptional rates (Figure 2), we do not find any special enrichment for Hmt1 near the 3'-end of the gene body (Figure 3A). Given the preferred Hmt1 occupancy at an average tDNA gene, it implicates a potential role for this enzyme in modulating tRNA biogenesis, potentially either at the level of transcription or via processing, as transcribed tRNAs undergo many processing events such as base modification and splicing during their maturation.

Interestingly, we found that the majority of $\mathrm{Hmt} 1 \mathrm{occu}-$ pancy at tDNAs did not follow an all or none trend when we examined the binding of Hmt1 to the entire set of alleles for a specific tRNA gene species. For example, we found Hmt1 occupancy at five of seven tDNAs encoding tK(UUU). However, in some instances such as tDNAs that encode $\mathrm{tG}(\mathrm{CCC})$, we did find Hmt1 occupancy at both of the tDNA alleles. As a way to determine whether Hmt1binding may be correlated to the codon usage, we calculated the correlation coefficient for Hmt1-binding and specific codon usage. However, such analysis did not reveal any credible candidates (data not shown), suggesting that Hmt1-binding to these tDNA genes is likely a general phenomenon.

\section{Mutants lacking Hmt1 or its catalytic activity display increased tRNA abundance}

To determine if the observed enrichment of Hmt1binding around tRNA genes had any functional significance, we used RNA hybridization assays to compare the levels of steady-state tRNA abundances between wild-type and Hmt1 loss-of-function mutants. We checked the matured form of tRNA tG(CCC), which is encoded by two tDNA alleles, both of which were bound by Hmt1 based on our analysis. In both Hmt1 loss-of-function mutants, there is an approximately two-fold increase in the levels of mature $\mathrm{tG}(\mathrm{CCC}) \mathrm{tRNA}$ when compared to that of the wild-type (Figure 3B, tG(CCC) panel). We next checked another tRNA gene, tK(UUU), that had five out of seven tDNA alleles bound by Hmt1. In this case, an increase in tRNA abundance is still observed, but the magnitude of the increase is not as significant as that of $\mathrm{tG}(\mathrm{CCC})$ (Figure 3B).

During tRNA biogenesis, various processing steps must take place in order to generate final, mature tRNAs. Hmt1 has been previously implicated in mRNP dynamics and many of its substrates are mRNA processing factors. Thus, it is possible that the change in the tRNA abundance observed in the Hmt1 loss-of-function mutants may be due to a role for Hmt1 in the tRNA processing steps. To test this possibility, we used a previously published probe that would hybridize equally to both the pre-processed form and the mature form of tL(UAG), which had one out of
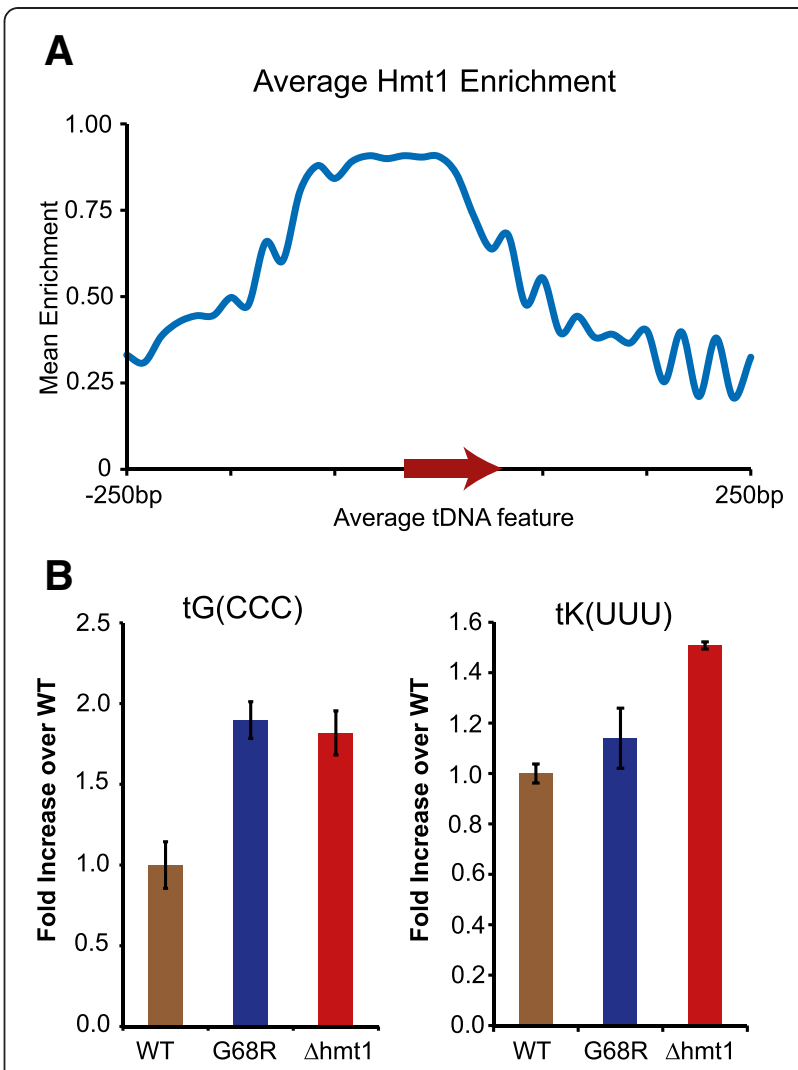

\section{C}

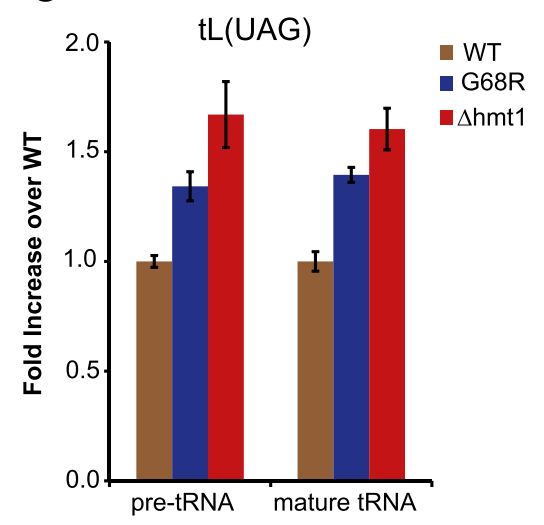

Figure $3 \mathrm{Hmt}$ 1-binding sites are enriched at tRNA genes. A) The average Hmt1-enrichment pattern across all Hmt1-bound tDNA features. B) Hmt1 loss-of-function mutants displayed increased tRNA abundance. Bar graph showing RNA hybridization analysis results on the relative abundance of mature tRNA tG(CCC) and tK(UUU) in the Hmt1-null ( $\Delta$ hmt1) and Hmt1-catalytically inactive mutants (G68R). Signals were normalized to the 5.8S rRNA (RNAPI transcript). C) Both pre- and mature tRNA abundance is increased in Hmt1 loss-offunction mutants. Bar graph showing RNA hybridization analysis results on the relative abundance of pre- and mature tRNA tL(UAG) in the Hmt1-null ( $\Delta \mathrm{hmt} 1)$ and Hmt1-catalytically inactive mutants (G68R). Signals were normalized to the 5.8S rRNA (RNAPI transcript). 
three tDNA alleles bound by Hmt1. In this case, we observe an increase in the pre-tRNA in the Hmt1 loss-of-function mutants, similar to that of the mature tRNA changes (Figure $3 \mathrm{C}$ ). Moreover, the ratio of pre-tRNA to total and the ratio of mature tRNA to total were not changed in these mutants. This comparison suggests that the increased tRNA abundance observed for the Hmtl loss-of-function mutants is likely due to a change in the overall transcriptional output of tRNA transcripts observed in these mutants.

\section{Hmt1 physically associates with TFIIIB component Bdp1}

Knowing that Hmt1 is bound at tRNA genes and that Hmt1 loss-of-function mutants display an increased tRNA abundance, there exists a potential regulatory role for Hmt1 in repressing RNAPIII transcription at tRNA genes. Given that the average enrichment of Hmt1 is mostly at the 5'-end and throughout the gene body, it is possible that Hmt1 may exert its control on tRNA biogenesis through some action on the components of the RNAPIII machinery. Interestingly, a recent proteomic profiling study for Hmt1 in our laboratory had identified the TFIIIB component Bdp1 as a potential physical interactor of Hmt1 (data not shown). Bdp1 is one of the three components of TFIIIB [43] and has been implicated to function as a scaffold for TFIIIB-DNA assembly [44]. To verify this physical association between TAP-tagged Hmt1 and Bdp1, we performed a co-immunoprecipitation with a reversed bait and target. In this case, we purified TAP-tagged Bdp1 and the purified eluates were assayed for the presence of myc-tagged Hmt1 by immunoblotting. Our immunoblotting data (Figure 4A) show that purification of TAP-tagged Bdp1 results in co-purification of Hmt1. As a negative control, we have carried out a parallel purification using lysates from a yeast strain that lack TAP-tagged proteins. Thus, we have confirmed the physical association between TFIIIB component Bdp1 and that of Hmt1.

It is likely that the Hmt1 recruitment to tRNA genes occurs via its physical association with Bdp1. While our ChIP-chip data only show Hmt1-binding to approximately $40 \%$ of all tDNAs, the observation of Hmt1's physical association with Bdp1 suggests that Hmt1 is binding to the majority of the tDNAs, if not all of them. This is based on the fact that TFIIIB occupancy is found across all tDNAs from a number of genome-wide location analyses in S. cerevisiae [45-47]. Therefore, it is likely that our stringent criteria for classifying what is considered an Hmt1bound locus excludes some true Hmt1-bound sites at these tDNAs. To test this hypothesis, we used ChIP to determine the association of $\mathrm{Hmt} 1$ at four different tDNAs, which were not bound by Hmt1 based on our ChIP-chip data. As shown in Figure 4B, however, there is an enrichment of Hmt1 at all four tDNAs examined, suggesting that indeed there may be some Hmt1-binding
A

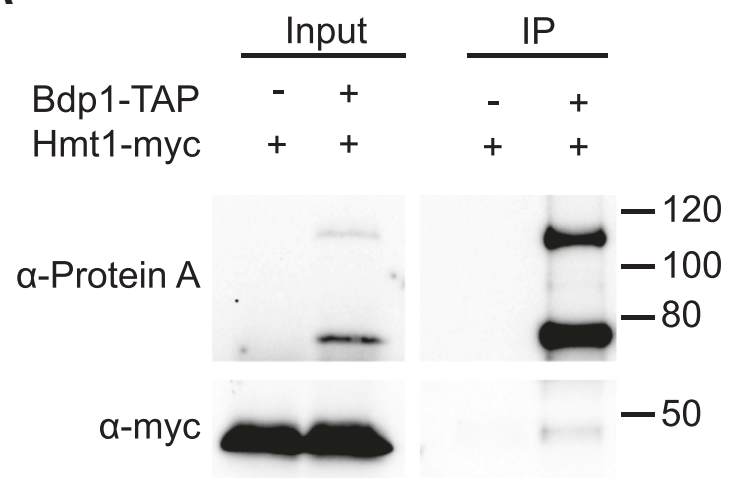

B

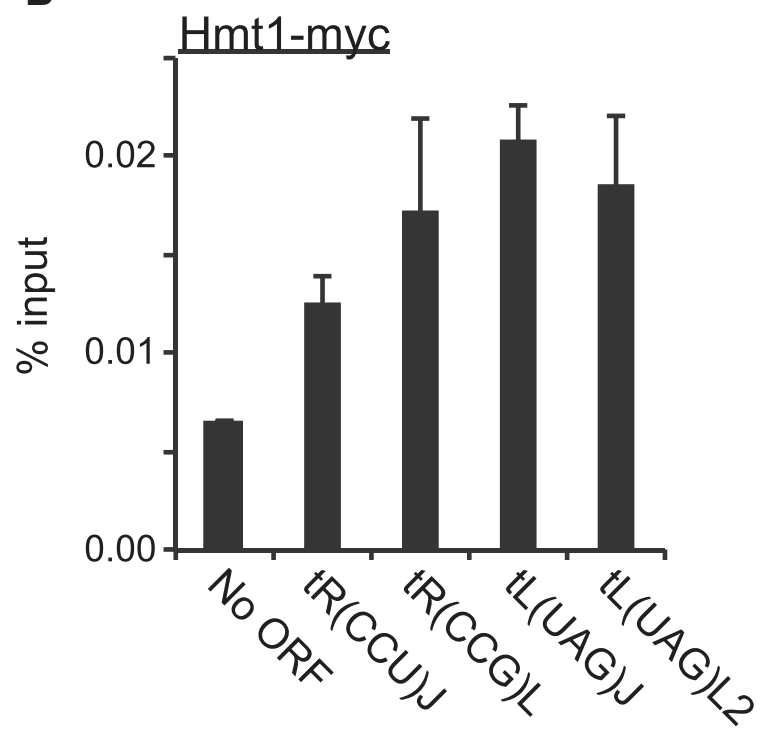

Figure 4 Hmt 1 physically associates with TFIIIB component Bdp1. A) Affinity purification of Bdp1-TAP results in the Copurification of Hmt1-myc, as shown by immunoblotting with a-myc (for Hmt1) and a-Protein A (for Bdp1). B) Directed ChIP shows Hmt1 binding on tDNAs tR(CCU)J, tR(CCG)L, tL(UAG)J, tL(UAG)L2. ChIP signals were normalized to "No ORF", which is an intergenic region on the right arm of Chromosome IV [68].

sites that are excluded in our final ChIP-chip data set because of high stringency. This also means that we are highly confident of the Hmt1-binding sites determined from our original analysis.

\section{Discussion}

In this study, we applied genome-wide location analysis to uncover genomic features previously not known to associate with Hmt1, the yeast homolog of human PRMT1. To this end, we identified binding of Hmt1 to a number of ncRNA genes such as tRNA and snoRNA genes. Enrichment of Hmt1 binding at these genomic features directly implicates a regulatory role for this protein in biological processes or pathways involving these 
genomic features. We validated the biological significance of Hmt1 binding at the tRNA genes using RNA hybridization analyses in assaying Hmt1 loss-of-function mutants, which display an increase in the steady-state levels of pre- and mature tRNA when compared to the wild-type cells. This increase is likely attributed to a change in the overall transcriptional output by RNAPIII, as opposed to a defect in the maturation process of tRNAs. The physical association between Hmt1 and TFIIIB component Bdp1 provides a likely explanation for Hmt1's association at many of the tRNA genes in our ChIP-chip data.

Hmt1 has previously been demonstrated to play a role in the recruitment of mRNP components such as premRNA splicing factors [48] and mRNA export factors [9]. Furthermore, it regulates transcriptional elongation and termination via methylation of hnRNP and mRNA export factor Npl3 [49]. Thus, it was not surprising for us to find the majority of Hmt1 binding sites fall proximal to or within an ORF. GO-enrichment analysis indicates Hmt1 binding to genes involved in translation, suggesting a potential role for this enzyme in modulating the expression of these genes. Transcriptional profiling experiments carried out previously on both null and catalytically-inactive mutants of Hmt1, however, show no significant changes in the abundance of these transcripts from the wild-type cells [9]. Based on these observations, one possible explanation is that Hmtl's effects on these genes can only be seen under a specific circumstance, such as a change in the environmental condition or an exposure to certain stress agent. This scenario would support the observed growth phenotype for the Hmt1-null mutants as they display no distinguishable changes in their growth rate from wild-type cells when grown in a rich media, such as YPD [6]. Transcription of HMT1 is repressed in response to heat shock when compared to steady-state growth rate conditions [50], further suggesting that Hmt1 is needed the most only under a certain growth condition. It is interesting to note that Hmt1 has different occupancy patterns across genes with different transcriptional rates, as this observation demonstrates a potential for different modes of regulation during gene expression by Hmt1. There are two functional implications of Hmt1's enrichment across the various transcriptional frequency gene classes - first, the highly transcribed gene class contains many of the ribosomal protein genes that encompass many introncontaining genes and Hmt1 has been shown to be more enriched at the 5'-end of these genes. Since Hmt1 affects the co-transcriptional recruitment of pre-mRNA splicing factors [10], Hmt1's enrichment across this region may reflect its role in regulating this process. Second, Hmt1's enrichment across all ORFs shows a "spike" at the 3'-end of the genes, with this trend more pronounced in gene classes with medium and low transcriptional frequencies. This enrichment trend may reflect the association of Hmt1 with known substrates involved in 3'-end processing of a transcript, such as Yra1, Hrp1, and Nab2. Thus, these observations suggest that Hmt1 may methylate its substrate during transcription rather than prior to.

Hmt1 methylates H4R3 in vitro [7] and loss-of-function mutants of Hmt1 display decreased dimethylated H4R3 levels across silent chromatin regions [14], despite the observation that bulk dimethylated H4R3 is unchanged in hmt1 mutants [7]. Thus, Hmt1-catalyzed H4R3me2 in $S$. cerevisiae is associated with gene silencing. H4R3 methylation by PRMT1 in vivo is required for many subsequent histone modifications in mammalian cells [30]. It can be inferred then that Hmt1 may also play a role in modulating the levels of other histone modifications. By determining the enrichment or depletion of core histones and other histone modifications for Hmt1-bound ORFs, the data reveal that Hmt1 may coordinate regulation at the promoter with $\mathrm{H} 3$-specific acetyltransferases such as SAGA or NuaA3 and the Rpd3(S) complex, which is an H3K36 methyl reader [51-53]. Furthermore, our ORFbody analysis suggests that Hmt1 may coordinate the action of COMPASS (responsible for H3K4 methylation), Set2 (responsible for H3K36 methylation) as well as KDACs [54-57]. Results from our directed ChIP experiments, however, suggests that these histone modification marks may regulate the recruitment of Hmt1 to ORFcontaining gene bodies instead, at least for pan-H4 acetylation and the majority of $\mathrm{H} 4 \mathrm{~K} 36 \mathrm{me} 2$ tested. It is possible that the loss of Hmt1 alone is not sufficient to alter these histone modification states we have identified. Rather, it may be the combination of Hmt1-deletion with the loss of another regulator that effects a significant change in histone modification levels as compared to any single lossof-function mutant.

Perhaps the most interesting hypothesis we obtained from analyzing our ChIP-chip data is a role for Hmt1 in the biogenesis of tRNAs. In eukaryotic cells, tRNAs are classified on the basis of their anticodon, of which there are 42 different tRNA species in the $S$. cerevisiae $[58,59]$. Furthermore, the actual number of different tRNA species is greater in some cases because there can be species with identical anticodons but sequence differences in the tRNA body [60]. In $S$. cerevisiae, each tRNA species can be encoded by several genomic copies (tDNAs), of which there are a total of 274 nuclear-encoded tDNAs within the genome [59]. Hmt1 binding is enriched at many of these tDNA alleles and mutants lacking Hmt1 or its catalytic activity display higher levels of the corresponding tRNA abundance. This overall change is likely attributed to a defect in the output rather than the processing during tRNA biogenesis, as both pre- and mature tRNAs increase in a similar fashion in the Hmt1 loss-of-function mutants. 
If the change in the mature tRNA abundance was attributed to a defect in tRNA processing in the Hmt1 mutants, we would expect the ratio (pre- to mature tRNA) of signals obtained from our RNA hybridization analysis to reflect such.

Transcription of tRNAs is accomplished by RNAPIII and this process requires two gene-internal promoters, boxA and boxB reviewed in [61,62]. During tRNA gene transcription, these internal promoters are bound by the hetero-heptameric complex TFIIIC. TFIIIC recruits the hetero-trimeric TFIIIB (which consists of Bdp1, Brf1, and TBP) to the upstream region of the tDNA. TFIIIB, in turn, recruits RNAPIII to prompt transcriptional initiation. The physical interaction between Hmt1 and Bdp1 provides a probable mechanism for how Hmt1 may be recruited to these tDNAs. Additionally, this physical interaction gave insights into how Hmt1 may affect the overall tRNA abundance through its ability to regulate RNAPIII transcription of tRNA genes. While the precise molecular mechanisms by which Hmt1 accomplishes this remains to be elucidated, our data provide a valuable clue in that Hmt1 does so via its catalytic activity, based on our data from the Hmt1 catalytically inactive mutant. This points to a yet-to-be discovered substrate of Hmt1 in which its methylation may affect the transcription of tRNA genes by RNAPIII. The biological significance of this transcriptional repression by Hmt1 could reflect a way for coordinating gene expression between RNAPII and RNAPIII, given the known role of Hmt1 in coordinating mRNP biogenesis catalyzed by RNAPII. As it is advantageous for cells to efficiently utilize its resources during growth (especially in sub-optimal conditions), proper coordination would be important for not wasting any valuable resources in executing gene expression programs. Thus, it can be inferred that Hmt1's function in balancing these gene expression programs may not be as critical when cells are growing under optimal conditions with plenty of resources, as supported by the observation that hmt1null cells do not display any obvious growth defect in rich media. Further experiments are required to define the specific molecular mechanisms used by Hmt1 in this process as well as other roles Hmtl may play in the described occupancy relationship for genomic features found within Hmt1 binding sites. In sum, our genomewide location analysis data do reveal multiple potentially new roles for Hmt1 in the control of eukaryotic gene expression in vivo, especially in the realm of ncRNA biogenesis.

\section{Conclusions}

Our data on the comprehensive mapping of Hmt1 binding sites across the yeast genome uncover many genomic features previously not known to associate with Hmt1, thereby implicating novel role of this enzyme in modulating biological processes facilitated by these genomic features. One such example we validated in this study is the tRNA gene, for which Hmt1 occupancy is enriched. The functional implication of Hmt1 binding on tRNA biogenesis is supported by the observation that binding of Hmt1 at these tRNA genes correlates with their abundance levels within a cell. At the molecular level, our data suggest that Hmt1 is recruited by Bdp1, a component of RNAPIII transcriptional machinery, to the tRNA genes. Together, these results suggest a potential role for Hmt1 in modulating tRNA biogenesis via RNAPIII transcription. Overall, our study demonstrates that knowledge of genome-wide locations for a multi-functional protein such as Hmt1 has the potential to lay the foundation for studies that reveal the factors responsible for recruiting $\mathrm{Hmt} 1$ to a specific genomic feature. Given the level of conservation between Hmt1 and human PRMT1, it is likely that some of our observations have implications for studies in higher eukaryotes.

\section{Methods}

Yeast strains used in this study

All yeast Saccharomyces cerevisiae strains used in this study are listed in Table 2 of the section. All strains were grown at $30^{\circ} \mathrm{C}$ on YEPD medium (1\% yeast extract, $2 \%$ bactopeptone, $2 \%$ D-glucose, w/v) unless otherwise stated

\section{Hmt1 ChIP-chip experiment}

The ChIP-chip procedure was performed as described previously [9]. Briefly, formaldehyde was used to crosslink myc-tagged Hmt1 and associated nucleic acids in vegetatively growing yeast cells. Upon harvesting of the crosslinked yeast cells, soluble chromatin was prepared by sonication to generate chromatin fragments on an average of $300 \mathrm{bp}$. Following sonication, the chromatin was subjected to immunoprecipitation using 9E11 anti-myc antibody (ThermoFisher) to enrich for Hmt1-associated DNA. After reversal of the crosslinks, the enriched DNA was amplified linearly by ligation-mediated PCR. As a control, a sample was prepared from total DNA (without undergoing immunoprecipitation). The resulting, enriched DNA samples were sent to NimbleGen for hybridization to highresolution $S$. cerevisiae tiling arrays (385K arrays), followed by the scanning and pre-processing of the array data. All the microarray data were deposited in NCBI's GEO database, Accession: GSE40505

For data analysis, the "Algorithm for Capturing Microarray Enrichment" (ACME) [63] was used to determine the sequence regions that were bound by epitope-tagged Hmt1 on the chromosome. ACME, which does not assume that $\log _{2}$ ratio data are Gaussian-distributed like other 
Table 2 Yeast strains used

\begin{tabular}{|c|c|c|}
\hline Strain \# & Genotype & Ref \\
\hline MYY65 & ade2-1 trp1-1 can1-100 leu2-3,112 his3-11,15 ura3 GAL+ psi+ HMT1::9MYC::TRP & [9] \\
\hline BY4741 & his $3 \Delta 1$ leu2 $\Delta 0$ met $15 \Delta 0$ ura3 $\Delta 0$ & [70] \\
\hline MYY649 & his3 1 leu2 $\triangle 0$ met15 $\Delta 0$ ura3 $\triangle 0 \Delta$ hmt1::KANMX6 hmt1-G68R::LEU2 & This Work \\
\hline MYY432 & his3 $\triangle 1$ leu2 $\triangle 0$ met15 $\triangle 0$ ura3 $\triangle 0 \Delta$ mmt1::KANMX6 & This Work \\
\hline MYY1200 & 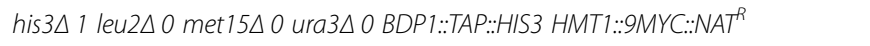 & This Work \\
\hline MYY1097 & his $3 \Delta 1$ leu2 $\triangle 0$ met15 $\triangle 0$ ura3 $\triangle 0$ HMT1::9myc::NATR & This Work \\
\hline
\end{tabular}

algorithms, makes only two assumptions: (1) that data are enriched for signal in the positive direction ("one-tailed") and (2) that the real signal will be represented by multiple probes that are genomically located close to one another ("neighbor effect"). Despite its simplicity, ACME has been proven to be quite robust at detecting true signal from noise [64]. For our analysis, the raw array data was normalized using bi-weight mean by NimbleScan Version 2.1 software (NimbleGen Systems).

To identify potential sites of enrichment, multiple sliding windows of 600-, 800- and 1,000-bp were separately employed to move stepwise along the tiled region, centering at every probe. Hybridization signals of probes within each window were tested by $x^{2}$ analysis to determine if the window contains a higher than expected number of probe signals above the defined threshold. The resulting $p$-value that is associated with each averaged data point was used for the selection of significant regions. To minimize false positives, we also employed a two-state hidden Markov model in an independent parallel analysis of the data set [65], in which two distributions were constructed to characterize binding and non-binding probe intensities, to determine regions of significance. The common significant regions from the above two methods were considered to be true signals. The three biological replicate sets of Hmt1 binding site data generated were then coalesced by assessing the binding-site midpoint distances and merging peaks from at least two datasets that were less than $125 \mathrm{bp}$ apart. Peaks were then either extended or truncated to $250 \mathrm{bp}$ (midpoint + /- $125 \mathrm{bp}$ ) for uniformity.

\section{Bioinformatics analysis of Hmt1 binding sites}

Annotations were done using PERL scripts and a dump of the SGD genome database feature file (downloaded on 09/ 2010). Average feature plots were generated as previously described [29]. Briefly, ORF sets were partitioned into 50 bins and the probes were summed and averaged for each bin. To minimize sampling errors, the average bin size for a class of ORFs was used to create bins in the upstream and downstream regions, which were fixed at $250 \mathrm{bp}$. For motif searches, Hmt1-binding sequences were first masked (A.F.A. Smit, R. Hubley \& P. Green, unpublished data. Current Version: open-3.3.0) and then analyzed using MEME [33], AlignACE [32], Weeder [34], and MDScan [35] with their default settings.

Histone modification enrichment/depletion analysis was carried out at the ChromatinDB website (http://www.bioinformatics2.wsu.edu/cgibin/ChromatinDB/cgi/visualize_select.pl) [31]. The input list of Hmt1-bound ORFs was used for nucleosome occupancy-normalized histone modifications, with a $p$-value cutoff of 0.001 , and Bonferroni multiple hypothesis test correction [31].

To search whether the identified Hmt1 binding sites are enriched for other transcription factor (TF) binding sites, we preformed the following analysis: A size of $250 \mathrm{bp}$ is assigned for each peak and used to determine which TF binds the peak. The CLOVER program was used with 184 fungi position weight matrixes (PWMs) from public domain information on TFs [66] to predict TF binding sites. Parameters of CLOVER [67] were set for 1000 randomizations and a $p$-value threshold of 0.05 using background sequences of 10,000 random DNA fragments of the same size generated from yeast chromosomes 1, 3, 5, 7, and 9 (2000 fragments from each chromosome).

\section{Chromatin immunoprecipitation (ChIP)}

ChIP procedures were performed as described previously [68] with at least duplicate biological samples (Hmt1-myc; $\mathrm{n}=2$, pan-acetyl-H4 and H3K36me2; n=3). For each biological replicate, qPCR was performed in three technical replicates. For qPCR, $2 \mu \mathrm{l}$ of DNA sample (input or IP) was used in $20 \mu \mathrm{l}$ reactions with $250-500 \mathrm{nM}$ final concentration of each primer and Bio-Rad Sso Advanced SYBR Green Supermix or Invitrogen Power SYBR Green PCR Master Mix. Table 3 shows the primers used in all qPCR experiments in this study. For each immunoprecipitation, $10 \mu \mathrm{l}$ of monoclonal $\alpha$-myc (9E11, Thermo-Fisher), or $5 \mu \mathrm{l}$ of $\alpha$-H3K36me2 (EMD Millipore cat\#07-369), or $5 \mu \mathrm{l}$ of $\alpha$-pan-acetyl-H4 (Upstate Biotechnology, cat\#06-598) was pre-coupled to $40 \mu \mathrm{l}$ of Protein-A sepharose beads.

\section{RNA hybridization analysis}

Total RNA was prepared from yeast strains (Table 2) grown to early log phase $\left(\mathrm{OD}_{600}=0.2\right.$ to 0.3$)$, using the hot phenol method as previously described [69]. For RNA hybridization analysis, $20 \mu \mathrm{g}$ of total RNA was resolved on 
Table 3 Oligonucleotides used in this study

\begin{tabular}{|c|c|c|}
\hline NAME & Sequence $\left(5^{\prime} \rightarrow 3^{\prime}\right)$ & Reference \\
\hline 5' -PMA1(sense) & CGACGACGAAGACAGT GATAACG & This study \\
\hline 5' -PMA1(anti-sense) & ATTGAATTGGACCGACGAAAAACAT AAC & This study \\
\hline 3' -PMA1 (sense) & AAA CAA CCA GCT TCG GTG TGT GTG & This study \\
\hline 3' -PMA1 (anti-sense) & TAG GAG CCA ACA AGA ATA AGC CGC & This study \\
\hline ILV5 (sense) & ATT GGG TAC CAG TCC AAA GCA CCT & This study \\
\hline ILV5 (anti-sense) & ACC GTC GAA GAA GCT ACC CAA TCT & This study \\
\hline RPS25A (sense) & TGG GTA GCT AGA TCT GTG CTA TGG & This study \\
\hline RPS25A (anti-sense) & GAA ATA GTG ACT GGC CAA TCC AGG & This study \\
\hline GAL1 (sense) & TGCTAGATCGCCTGGTAGAG & {$[72]$} \\
\hline GAL1 (anti-sense) & GCAAACCTTTCCGGTGCAAG & [72] \\
\hline MND1 (sense) & CCATCATGGTACACAGGACTGAACA & This study \\
\hline MND1 (anti-sense) & GGAGTGATACTGTCTGTCTCTITGGC & This study \\
\hline BET4 (sense) & CGACAGCAAGTTCAGTTTGCCACA & This study \\
\hline BET4 (anti-sense) & AGCGAGTTTGGCTTCTGAGTTGGA & This study \\
\hline SEC31 (sense) & CCAAATGATATCGCCCAGAGGAATG & This study \\
\hline SEC31 (anti-sense) & CCGGCAGCCAAGTACAAAGTCAAA & This study \\
\hline RPS13A (sense) & CAGAAAGCACTGGCAAGAACGTGT & This study \\
\hline RPS13A (anti-sense) & TCTGCCAGCTCTGACCTTTCTGTT & This study \\
\hline RPS14A (sense) & TGGTAAGGAAACCATCGCCAGAGT & This study \\
\hline RPS14A (anti-sense) & TAACGTGAACGGCAGTGATACCGA & This study \\
\hline YLL011W (sense) & AGCCAGTTTCCGATCTATCATGGG & This study \\
\hline YLL011W (anti-sense) & GGCCTCCATTGGATTCCAGCAAAT & This study \\
\hline snR37 (sense) & TGGAGTGTGAGTGATGAGGAGCTT & This study \\
\hline snR37 (anti-sense) & GGAGTAGTCAAAGTTCATTCAGCTATGGG & This study \\
\hline snR42 (sense) & CTGTTGGTGCTGAGGTAATCCATC & This study \\
\hline snR42 (anti-sense) & ACCTCAGGTCATCACCATTTCATGGG & This study \\
\hline ACT1 5' end (sense) & GTCCCAATTGCTCGAGAGATTTCTC & This study \\
\hline ACT1 5' end(anti-sense) & CATGATACCTTGGTGTCTTGGTC & This study \\
\hline ACT1 3' end(sense) & TCGAACAAGAAATGCAAACCG & This study \\
\hline ACT1 3' end(anti-sense) & GGCAGATTCCAAACCCAAAAC & This study \\
\hline CDC2 (sense) & CCTGCCTITAAGGCTTATGGA & This study \\
\hline CDC2 (anti-sense) & CCACGAATAGGCTCAATAACA & This study \\
\hline TRA1 (sense) & CCAATTITGATAAGCCACCCTGA & This study \\
\hline TRA1 (anti-sense) & CGTAATTTCTAAGGTCTTGTTCTCCCA & This study \\
\hline SPS2 (sense) & ACTGTCCCGTCATTGATGCGTCTC & [73] \\
\hline SPS2 (anti-sense) & GGGATCGTTGCATTAGTGTTAACC & [73] \\
\hline No ORF (sense) & GAAAAAGTGGGATTCTGCCTGTGG & {$[68]$} \\
\hline No ORF (anti-sense) & GTTGCCACAGCGACAGAAGTATAACC & {$[68]$} \\
\hline tG (CCC) D (sense) & AAATGCGGAAGCCGGGAATCGAA & This study \\
\hline tG (CCC) D (anti-sense) & GGTATTTCTTTGCGCGGTTACGGT & This study \\
\hline tL (UAG) L1 (sense) & GGT TAAACCCAC CTAAATCTGACGCC & This study \\
\hline tL (UAG) L1 (anti-sense) & TGGCTAACCCAATGGCTTGT & This study \\
\hline tW (CCA) P (sense) & AAGCTGAGTGTCCGCTGTGATGAT & This study \\
\hline tW (CCA) P (anti-sense) & TTGCAATCGAAGGGTTGCAGGTTC & This study \\
\hline
\end{tabular}


Table 3 Oligonucleotides used in this study (Continued)

\begin{tabular}{|c|c|c|}
\hline$\overline{\mathrm{tK}}(\mathrm{UUU}) \mathrm{D}$ (sense) & TGTTGGAACGGTAAAGACCAGTGC & This study \\
\hline tK (UUU) D (anti-sense) & AAAGCCGAACGCTCTACCAACTGA & This study \\
\hline $\mathrm{tG}(\mathrm{CCC})$ probe & ATGCTTGGGAAGCATAAATTCTA & This study \\
\hline tL (UAG) probe & AAGATATCAGAGCCTAAATCTGACG & [71] \\
\hline pre-t L(UAG) probe & GATATCAGAGATTTTAGAGGTTAAATCCACCT & [71] \\
\hline 5.85 probe & GCGTTGTTCATCGATGC & This study \\
\hline tK (UUU) probe & TGACATTTCGGTTAAAAGCCGAACGCTCTACCAAC & [71] \\
\hline tR (CCG) L (sense) & AAGTACGACATCAAAGTCGCCGAG & This study \\
\hline tR (CCG) L (anti-sense) & TGCTAACCATTGCACTAGAGGAGC & This study \\
\hline snR128 (sense) & ACAGTATACGATCACTCAGACATCC & This study \\
\hline snR128 (anti-sense) & CACGGTGATGAAAGACTGGTTCCT & This study \\
\hline tR (CCU) L (sense) & CCACATTCTCTACAATATTGATTTCCATCG & This study \\
\hline tR (CCU) L (anti-sense) & CCATTACGCCAACGGAACCAACTT & This study \\
\hline tL (UAG) L2 (sense) & ATCGACAGCTTCACGTGCCATTTG & This study \\
\hline tL (UAG) L2 (anti-sense) & CTGATATCTTCGGATGCAAGGGTTCG & This study \\
\hline tL (UAG) J (sense) & GGTTAAACCCACCTAAATCTGACGCC & This study \\
\hline tL (UAG) J (anti-sense) & TGGCTAACCCAATGGCTTGT & This study \\
\hline
\end{tabular}

an $8 \%$ PAGE-urea (7.5M) gels and then transferred to HybondN+ Nylon membranes (GE Healthcare) using Bio-Rad semi-dry transfer (150 mA for $1 \mathrm{hr}$ ). The membranes were then UV-crosslinked with a Stratalinker using the Optimal Crosslink setting. Membranes were hybridized in 3X SSC, 1X Denhardt's, 0.5\% SDS, and 100 $\mu \mathrm{g} / \mathrm{mL}$ sheared salmon sperm DNA overnight at $37^{\circ} \mathrm{C}$ with ${ }^{32} \mathrm{P}$-end labeled oligonucleotide probes (see Table 3 for sequences). After hybridization, membranes were rinsed once and then washed twice for 20 mins with $50 \mathrm{ml}$ of $2 \mathrm{X} \mathrm{SSC} / 0.1 \% \mathrm{SDS}$ buffer at $37^{\circ} \mathrm{C}$. The membranes were exposed to PhosphorStorage screens, scanned with a PhosphorImager (Molecular Dynamics) and quantified using the ImageQuant software.

\section{Coimmunoprecipitation study between Hmt1 and Bdp1}

Preparation of yeast cell lysates for co-immunoprecipitations between Hmt1-myc and Bdp1-TAP was carried out as described previously [48]. Bdp1-TAP was purified by incubating prepared yeast cell lysates with $40 \mu \mathrm{L}$ IgG sepharose beads (GE-Amersham) for 2 hours at $4^{\circ} \mathrm{C}$, followed by three washes with IPP150 buffer. Bound protein was eluted in $300 \mu \mathrm{L}$ Elution Buffer (0.5 M Acetic Acid pH 3.5), precipitated with trichloroacetic acid (TCA) overnight at $-20^{\circ} \mathrm{C}$, recovered, and resolved on a NuPAGE 4-12\% Bis-Tris gradient gel (Life Technologies). The immunoblot was probed with an anti-myc antibody (Santa Cruz A-14), stripped, and re-probed with an of the immunoblot $\alpha$-Protein A antibody (Sigma, cat\#P3775).

\section{Additional files}

Additional file 1: Table S1. Hmt1 binding site coordinates trimmed to $250 \mathrm{bp}$. The file is in gff format, the columns are listed below: Column 1: Chromosome. Column 2: Source. Column 3: feature. Column 4: Start Coordinate. Column 5: Stop Coordinate. Column 6: Score. Column 7: Strand. Column 8: Reading Frame. Column 9: Attributes

Additional file 2: Table S2. TF motif search. Results from transcription factor motif search. Transcription factors are listed on left, followed by number of Hmt 1 peaks found to contain the TF's motif. Followed by three different methods of background motif estimation. 1) Sequence shuffle of Hmt1 peaks, 2) S. cerevisiae promoter sequences (-500bp to +100bp of every ORF) and 3) 3'-UTR sequences for each ORF.

\section{Abbreviations}

ACME: Algorithm for Capturing Microarray Enrichment; ADMA: Asymmetric dimethylarginine; ARS: Autonomously replicating sequence; MMA: Monomethylarginine; PRMT: Protein arginine methyltransferase; RNAPI: RNA polymerase I; RNAPII: RNA polymerase II; RNAPIII: RNA polymerase III; SDMA: Symmetric dimethylarginine; TF: Transcription factor.

\section{Competing interests}

The authors declare that they have no competing interests.

\section{Authors' contributions}

EJM and MCY conceived and designed the experiments. MCY carried out the ChIP-chip experiments. EJM and ZH performed and contributed to the microarray data processing and analysis. EJM carried out additional experiments and data analysis. EJM and MCY wrote the paper. All authors read and approved the final manuscript.

\section{Acknowledgements}

We thank Michael Buck and members of the Yu lab for helpful discussions and critical reading of the manuscript. This work was supported by a Scientist Development Grant (0830279N) from the American Heart Association and a National Science Foundation award (Award ID\#1051350) to MCY. The funders had no role in study design, data collection and interpretation, decision for preparation and publishing of the manuscript. 


\section{Author details}

${ }^{1}$ Department of Biological Sciences, State University of New York at Buffalo, Buffalo, NY 14260, USA. ${ }^{2}$ Center for Computational Research, New York State Center of Excellence in Bioinformatics \& Life Sciences, Department of Ophthalmology, Department of Biostatistics, Department of Medicine, State University of New York at Buffalo, Buffalo, NY 14260, USA. ${ }^{3}$ SUNY Eye Institute, Buffalo, NY 14260, USA

Received: 18 September 2012 Accepted: 21 December 2012

Published: 26 December 2012

\section{References}

1. Bedford MT, Richard S: Arginine methylation an emerging regulatorof protein function. Mol Cell 2005, 18(3):263-272

2. Bachand F: Protein arginine methyltransferases: from unicellular eukaryotes to humans. Eukaryot Cell 2007, 6(6):889-898.

3. Niewmierzycka A, Clarke S: S-Adenosylmethionine-dependent methylation in Saccharomyces cerevisiae. Identification of a novel protein arginine methyltransferase. J Biol Chem 1999, 274(2):814-824.

4. Bedford MT, Clarke SG: Protein arginine methylation in mammals: who, what, and why. Mol Cell 2009, 33(1):1-13.

5. Henry MF, Silver PA: A novel methyltransferase (Hmt1p) modifies poly(A) +-RNA-binding proteins. Mol Cell Biol 1996, 16(7):3668-3678.

6. Gary JD, Lin WJ, Yang MC, Herschman HR, Clarke S: The predominant protein-arginine methyltransferase from Saccharomyces cerevisiae. J Biol Chem 1996, 271(21):12585-12594.

7. Lacoste N, Utley RT, Hunter JM, Poirier GG, Cote J: Disruptor of telomeric silencing-1 is a chromatin-specific histone $\mathrm{H} 3$ methyltransferase. J Biol Chem 2002, 277(34):30421-30424.

8. Miranda TB, Sayegh J, Frankel A, Katz JE, Miranda M, Clarke S: Yeast HsI7 (histone synthetic lethal 7) catalyses the in vitro formation of omega-N (G)-monomethylarginine in calf thymus histone H2A. Biochem J 2006, 395(3):563-570.

9. Yu MC, Bachand F, McBride AE, Komili S, Casolari JM, Silver PA: Arginine methyltransferase affects interactions and recruitment of mRNA processing and export factors. Genes Dev 2004, 18(16):2024-2035.

10. Chen YC, Milliman EJ, Goulet I, Cote J, Jackson CA, Vollbracht JA, Yu MC: Protein arginine methylation facilitates cotranscriptional recruitment of pre-mRNA splicing factors. Mol Cell Biol 2010, 30(21):5245-5256. doi:10.1128/MCB.00359-10. Epub 2010 Sep 7. PMID: 20823272.

11. Shen EC, Henry MF, Weiss VH, Valentini SR, Silver PA, Lee MS: Arginine methylation facilitates the nuclear export of hnRNP proteins. Genes Dev 1998, 12(5):679-691.

12. Green DM, Marfatia KA, Crafton EB, Zhang X, Cheng X, Corbett AH: Nab2p is required for poly(A) RNA export in Saccharomyces cerevisiae and is regulated by arginine methylation via Hmt1p. J Biol Chem 2002, 277(10):7752-7760.

13. Xu C, Henry PA, Setya A, Henry MF: In vivo analysis of nucleolar proteins modified by the yeast arginine methyltransferase Hmt1/Rmt1p. RNA (New York, NY 2003, 9(6):746-759.

14. Yu MC, Lamming DW, Eskin JA, Sinclair DA, Silver PA: The role of protein arginine methylation in the formation of silent chromatin. Genes Dev 2006, 20(23):3249-3254

15. Milliman EJ, Yadav N, Chen YC, Muddukrishna B, Karunanithi S, Yu MC: Recruitment of $\mathrm{rpd} 3$ to the telomere depends on the protein arginine methyltransferase hmt1. PLoS One 2012, 7(8):e44656.

16. Costanzo M, Baryshnikova A, Bellay J, Kim Y, Spear ED, Sevier CS, Ding H, Koh JL, Toufighi K, Mostafavi S, et al: The genetic landscape of a cell. Science (New York, NY 2010, 327(5964):425-431.

17. Wilmes GM, Bergkessel M, Bandyopadhyay S, Shales M, Braberg H, Cagney G, Collins SR, Whitworth GB, Kress TL, Weissman JS, et al: A genetic interaction map of RNA-processing factors reveals links between Sem1/ Dss1-containing complexes and mRNA export and splicing. Mol Cell 2008, 32(5):735-746.

18. Buck MJ, Lieb JD: ChIP-chip: considerations for the design, analysis, and application of genome-wide chromatin immunoprecipitation experiments. Genomics 2004, 83(3):349-360.

19. Bulyk ML: DNA microarray technologies for measuring protein-DNA interactions. Curr Opin Biotechnol 2006, 17(4):422-430.

20. Ren B, Robert F, Wyrick JJ, Aparicio O, Jennings EG, Simon I, Zeitlinger J, Schreiber J, Hannett N, Kanin E, et al: Genome-wide location and function of DNA binding proteins. Science (New York, NY 2000, 290(5500):2306-2309.

21. $\mathrm{Ng} \mathrm{HH}$, Robert F, Young RA, Struhl K: Genome-wide location and regulated recruitment of the RSC nucleosome-remodeling complex. Genes Dev 2002, 16(7):806-819.

22. Tardiff DF, Lacadie SA, Rosbash M: A genome-wide analysis indicates that yeast pre-mRNA splicing is predominantly posttranscriptional. Mol Cell 2006, 24(6):917-929.

23. Moore MJ, Schwartzfarb EM, Silver PA, Yu MC: Differential recruitment of the splicing machinery during transcription predicts genome-wide patterns of mRNA splicing. Mol Cell 2006, 24(6):903-915

24. Lieb JD, Liu X, Botstein D, Brown PO: Promoter-specific binding of Rap1 revealed by genome-wide maps of protein-DNA association. Nat Genet 2001, 28(4):327-334

25. Harbison CT, Gordon DB, Lee TI, Rinaldi NJ, Macisaac KD, Danford TW Hannett NM, Tagne JB, Reynolds DB, Yoo J, et al: Transcriptional regulatory code of a eukaryotic genome. Nature 2004, 431(7004):99-104

26. Jiang C, Pugh BF: Nucleosome positioning and gene regulation: advances through genomics. Nat Rev Genet 2009, 10(3):161-172.

27. Berriz GF, Beaver JE, Cenik C, Tasan M, Roth FP: Next generation software for functional trend analysis. Bioinformatics 2009, 25(22):3043-3044.

28. Holstege FC, Jennings EG, Wyrick JJ, Lee TI, Hengartner CJ, Green MR, Golub TR, Lander ES, Young RA: Dissecting the regulatory circuitry of a eukaryotic genome. Cell 1998, 95(5):717-728.

29. Pokholok DK, Harbison CT, Levine S, Cole M, Hannett NM, Lee TI, Bell GW Walker K, Rolfe PA, Herbolsheimer E, et al: Genome-wide map of nucleosome acetylation and methylation in yeast. Cell 2005, 122(4):517-527.

30. Huang S, Litt M, Felsenfeld G: Methylation of histone $\mathrm{H} 4$ by arginine methyltransferase PRMT1 is essential in vivo for many subsequent histone modifications. Genes Dev 2005, 19(16):1885-1893.

31. O'Connor TR, Wyrick JJ: ChromatinDB: a database of genome-wide histone modification patterns for Saccharomyces cerevisiae. Bioinformatics 2007, 23(14):1828-1830.

32. Roth FP, Hughes JD, Estep PW, Church GM: Finding DNA regulatory motifs within unaligned noncoding sequences clustered by whole-genome mRNA quantitation. Nat Biotechnol 1998, 16(10):939-945.

33. Bailey $T L$, Elkan C: The value of prior knowledge in discovering motifs with MEME. Proc Int Conf Intell Syst Mol Biol 1995, 3:21-29.

34. Pavesi G, Mauri G, Pesole G: An algorithm for finding signals of unknown length in DNA sequences. Bioinformatics 2001, 17(Suppl 1):S207-S214.

35. Liu XS, Brutlag DL, Liu JS: An algorithm for finding protein-DNA binding sites with applications to chromatin-immunoprecipitation microarray experiments. Nat Biotechnol 2002, 20(8):835-839.

36. Badis G, Chan ET, van Bakel H, Pena-Castillo L, Tillo D, Tsui K, Carlson CD, Gossett AJ, Hasinoff MJ, Warren $C L$, et al: A library of yeast transcription factor motifs reveals a widespread function for Rsc3 in targeting nucleosome exclusion at promoters. Mol Cell 2008, 32(6):878-887.

37. Maclsaac KD, Wang T, Gordon DB, Gifford DK, Stormo GD, Fraenkel E: An improved map of conserved regulatory sites for Saccharomyces cerevisiae. BMC Bioinformatics 2006, 7:113.

38. Cormack BP, Struhl $K$ : The TATA-binding protein is required for transcription by all three nuclear RNA polymerases in yeast cells. Cell 1992, 69(4):685-696.

39. Hedges D, Proft M, Entian KD: CAT8, a new zinc cluster-encoding gene necessary for derepression of gluconeogenic enzymes in the yeast Saccharomyces cerevisiae. Mol Cell Biol 1995, 15(4):1915-1922.

40. Bricmont PA, Daugherty JR, Cooper TG: The DAL81 gene product is required for induced expression of two differently regulated nitrogen catabolic genes in Saccharomyces cerevisiae. Mol Cell Biol 1991, 11(2):1161-1166.

41. Nehlin JO, Ronne H: Yeast MIG1 repressor is related to the mammalian early growth response and Wilms' tumour finger proteins. EMBO J 1990 9(9):2891-2898.

42. Strich R, Surosky RT, Steber C, Dubois E, Messenguy F, Esposito RE: UME6 is a key regulator of nitrogen repression and meiotic development. Genes Dev 1994, 8(7):796-810.

43. Kassavetis GA, Nguyen ST, Kobayashi R, Kumar A, Geiduschek EP, Pisano M: Cloning, expression, and function of TFC5, the gene encoding the $B^{\prime \prime}$ component of the Saccharomyces cerevisiae RNA polymerase III transcription factor TFIIIB. Proc Natl Acad Sci USA 1995, 92(21):9786-9790. 
44. Kumar A, Kassavetis GA, Geiduschek EP, Hambalko M, Brent CJ: Functional dissection of the $B^{\prime \prime}$ component of RNA polymerase III transcription factor IIIB: a scaffolding protein with multiple roles in assembly and initiation of transcription. Mol Cell Biol 1997, 17(4):1868-1880.

45. Harismendy O, Gendrel CG, Soularue P, Gidrol X, Sentenac A, Werner M, Lefebvre O: Genome-wide location of yeast RNA polymerase III transcription machinery. EMBO J 2003, 22(18):4738-4747.

46. Roberts DN, Stewart AJ, Huff JT, Cairns BR: The RNA polymerase III transcriptome revealed by genome-wide localization and activityoccupancy relationships. Proc Natl Acad Sci USA 2003, 100(25):14695-14700.

47. Moqtaderi Z, Struhl K: Genome-wide occupancy profile of the RNA polymerase III machinery in Saccharomyces cerevisiae reveals loci with incomplete transcription complexes. Mol Cell Biol 2004, 24(10):4118-4127.

48. Chen Y-C, Milliman EJ, Goulet I, Côté J, Jackson CA, Vollbracht JA, Yu MC: Protein arginine methylation facilitates cotranscriptional recruitment of pre-mRNA splicing factors. Mol Cell Biol 2010, 30(21):5245-5256.

49. Wong CM, Tang HM, Kong KY, Wong GW, Qiu H, Jin DY, Hinnebusch AG: Yeast arginine methyltransferase $\mathrm{Hmt} 1 \mathrm{p}$ regulates transcription elongation and termination by methylating Npl3p. Nucleic Acids Res 2010, 38(7):2217-2228

50. Lu C, Brauer MJ, Botstein D: Slow growth induces heat-shock resistance in normal and respiratory-deficient yeast. Mol Biol Cell 2009, 20(3):891-903.

51. Suka N, Suka Y, Carmen AA, Wu J, Grunstein M: Highly specific antibodies determine histone acetylation site usage in yeast heterochromatin and euchromatin. Mol Cell 2001, 8(2):473-479.

52. Grant PA, Duggan L, Cote J, Roberts SM, Brownell JE, Candau R, Ohba R, Owen-Hughes T, Allis CD, Winston F, et al: Yeast Gen5 functions in two multisubunit complexes to acetylate nucleosomal histones: characterization of an Ada complex and the SAGA (Spt/Ada) complex. Genes Dev 1997, 11(13):1640-1650.

53. Carrozza MJ, Li B, Florens L, Suganuma T, Swanson SK, Lee KK, Shia WJ, Anderson S, Yates J, Washburn MP, et al: Histone H3 methylation by Set2 directs deacetylation of coding regions by Rpd3S to suppress spurious intragenic transcription. Cell 2005, 123(4):581-592.

54. Miller T, Krogan NJ, Dover J, Erdjument-Bromage H, Tempst $P$, Johnston M, Greenblatt JF, Shilatifard A: COMPASS: a complex of proteins associated with a trithorax-related SET domain protein. Proc Natl Acad Sci USA 2001, 98(23):12902-12907.

55. Boa S, Coert C, Patterton HG: Saccharomyces cerevisiae Set1p is a methyltransferase specific for lysine 4 of histone $\mathrm{H} 3$ and is required for efficient gene expression. Yeast (Chichester, England) 2003, 20(9):827-835.

56. Strahl BD, Grant PA, Briggs SD, Sun ZW, Bone JR, Caldwell JA, Mollah S, Cook RG, Shabanowitz J, Hunt DF, et al: Set2 is a nucleosomal histone H3selective methyltransferase that mediates transcriptional repression. Mol Cell Biol 2002, 22(5):1298-1306.

57. Schaft D, Roguev A, Kotovic KM, Shevchenko A, Sarov M, Neugebauer KM Stewart AF: The histone 3 lysine 36 methyltransferase, SET2, is involved in transcriptional elongation. Nucleic Acids Res 2003, 31(10):2475-2482.

58. Cherry JM, Hong EL, Amundsen C, Balakrishnan R, Binkley G, Chan ET, Christie KR, Costanzo MC, Dwight SS, Engel SR, et al: Saccharomyces Genome Database: the genomics resource of budding yeast. Nucleic Acids Res 2012, 40(Database issue):D700-D705.

59. Cherry JM, Ball C, Weng S, Juvik G, Schmidt R, Adler C, Dunn B, Dwight S, Riles L, Mortimer RK, et al: Genetic and physical maps of Saccharomyces cerevisiae. Nature 1997, 387(6632 Suppl):67-73.

60. Goodenbour JM, Pan T: Diversity of tRNA genes in eukaryotes. Nucleic Acids Res 2006, 34(21):6137-6146.

61. Geiduschek EP, Kassavetis GA: The RNA polymerase III transcription apparatus. J Mol Biol 2001, 310(1):1-26.

62. Willis IM: RNA polymerase III. Genes, factors and transcriptional specificity. Eur J Biochem 1993, 212(1):1-11.

63. Scacheri PC, Crawford GE, Davis S: Statistics for ChIP-chip and DNase hypersensitivity experiments on NimbleGen arrays. Methods Enzymol 2006, 411:270-282

64. Scacheri PC, Davis S, Odom DT, Crawford GE, Perkins S, Halawi MJ, Agarwal SK, Marx SJ, Spiegel AM, Meltzer PS, et al: Genome-wide analysis of menin binding provides insights into MEN1 tumorigenesis. PLOS Genet 2006, 2(4):e51.

65. Ji H, Wong WH: TileMap: create chromosomal map of tiling array hybridizations. Bioinformatics 2005, 21(18):3629-3636.
66. Zhu C, Byers KJ, McCord RP, Shi Z, Berger MF, Newburger DE, Saulrieta K, Smith Z, Shah MV, Radhakrishnan M, et al: High-resolution DNA-binding specificity analysis of yeast transcription factors. Genome Res 2009, 19(4):556-566

67. Frith MC, Fu Y, Yu L, Chen JF, Hansen U, Weng Z: Detection of functional DNA motifs via statistical over-representation. Nucleic Acids Res 2004 32(4):1372-1381.

68. Lei EP, Silver PA: Intron status and 3/-end formation control cotranscriptional export of mRNA. Genes Dev 2002, 16(21):2761-2766.

69. Collart MA, Oliviero S: Preparation of yeast RNA. Curr Protoc Mol Biol 2001 Chapter 13: Unit13 12

70. Brachmann CB, Davies A, Cost GJ, Caputo E, Li J, Hieter P, Boeke JD: Designer deletion strains derived from Saccharomyces cerevisiae S288C: a useful set of strains and plasmids for PCR-mediated gene disruption and other applications. Yeast (Chichester, England) 1998, 14(2):115-132.

71. Copela LA, Fernandez CF, Sherrer RL, Wolin SL: Competition between the Rex 1 exonuclease and the La protein affects both Trf4p-mediated RNA quality control and pre-tRNA maturation. RNA (New York) 2008, 14(6):1214-1227.

72. Hoppe GJ, Tanny JC, Rudner AD, Gerber SA, Danaie S, Gygi SP, Moazed D: Steps in assembly of silent chromatin in yeast: Sir3-independent binding of a Sir2/Sir4 complex to silencers and role for Sir2-dependent deacetylation. Mol Cell Biol 2002, 22(12):4167-4180.

73. Xu F, Zhang Q, Zhang K, Xie W, Grunstein M: Sir2 deacetylates histone H3 lysine 56 to regulate telomeric heterochromatin structure in yeast. $\mathrm{Mol}$ Cell 2007, 27(6):890-900.

doi:10.1186/1471-2164-13-728

Cite this article as: Milliman et al:: Genomic insights of protein arginine methyltransferase $\mathrm{Hmt} 1$ binding reveals novel regulatory functions. BMC Genomics 2012 13:728.

\section{Submit your next manuscript to BioMed Central and take full advantage of:}

- Convenient online submission

- Thorough peer review

- No space constraints or color figure charges

- Immediate publication on acceptance

- Inclusion in PubMed, CAS, Scopus and Google Scholar

- Research which is freely available for redistribution 